\title{
Microbial ecology of an Antarctic hypersaline lake: genomic assessment of ecophysiology among dominant haloarchaea
}

\author{
Timothy J Williams ${ }^{1}$, Michelle A Allen ${ }^{1}$, Matthew Z DeMaere ${ }^{1}$, Nikos C Kyrpides ${ }^{2}$, \\ Susannah G Tringe ${ }^{2}$, Tanja Woyke ${ }^{2}$ and Ricardo Cavicchioli ${ }^{1}$ \\ ${ }^{1}$ School of Biotechnology and Biomolecular Sciences, The University of New South Wales, Sydney, \\ New South Wales, Australia and ${ }^{2}$ Department of Energy Joint Genome Institute, Walnut Creek, CA, USA
}

\begin{abstract}
Deep Lake in Antarctica is a cold, hypersaline system where four types of haloarchaea representing distinct genera comprise $>70 \%$ of the lake community: strain tADL $\sim 44 \%$, strain DL31 $\sim 18 \%$, Halorubrum lacusprofundi $\sim 10 \%$ and strain DL1 $\sim 0.3 \%$. By performing comparative genomics, growth substrate assays, and analyses of distribution by lake depth, size partitioning and lake nutrient composition, we were able to infer important metabolic traits and ecophysiological characteristics of the four Antarctic haloarchaea that contribute to their hierarchical persistence and coexistence in Deep Lake. tADL is characterized by a capacity for motility via flagella (archaella) and gas vesicles, a highly saccharolytic metabolism, a preference for glycerol, and photoheterotrophic growth. In contrast, DL31 has a metabolism specialized in processing proteins and peptides, and appears to prefer an association with particulate organic matter, while lacking the genomic potential for motility. $H$. lacusprofundi is the least specialized, displaying a genomic potential for the utilization of diverse organic substrates. The least abundant species, DL1, is characterized by a preference for catabolism of amino acids, and is the only one species that lacks genes needed for glycerol degradation. Despite the four haloarchaea being distributed throughout the water column, our analyses describe a range of distinctive features, including preferences for substrates that are indicative of ecological niche partitioning. The individual characteristics could be responsible for shaping the composition of the haloarchaeal community throughout the lake by enabling selection of ecotypes and maintaining sympatric speciation.

The ISME Journal (2014) 8, 1645-1658; doi:10.1038/ismej.2014.18; published online 20 February 2014

Subject Category: Integrated genomics and post-genomics approaches in microbial ecology

Keywords: genomics; Antarctic microbial ecology; nutrient cycles; ecophysiology; ecotype
\end{abstract}

\section{Introduction}

Deep Lake is a unique, cold, marine-derived hypersaline lake in Antarctica. It is the most hypersaline system in the Vestfold Hills, a permanently ice-free region on the eastern shore of Prydz Bay, East Antarctica, where over 300 lakes and ponds exist (Gibson, 1999; Wilkins et al., 2013). The temperature close to the surface of Deep Lake ranges from -16 to $12{ }^{\circ} \mathrm{C}$, with the temperature of the few meters at the lake surface exceeding $0{ }^{\circ} \mathrm{C}$ only for a few months of the year during the austral summer (Campbell, 1978; Wright and Burton, 1981; Ferris and Burton, 1988; Franzmann et al., 1988). Nevertheless, because of high salinity $\left(\sim 270 \mathrm{gl}^{-1}\right)$, the water column remains

Correspondence: R Cavicchioli, School of Biotechnology and Biomolecular Sciences, The University of New South Wales, Sydney, New South Wales 2052, Australia.

E-mail: r.cavicchioli@unsw.edu.au

Received 3 October 2013; revised 29 December 2013; accepted 17 January 2014; published online 20 February 2014 ice-free even in winter when the lake temperature falls to $-20^{\circ} \mathrm{C}$ (Campbell, 1978; Wright and Burton, 1981; Ferris and Burton, 1988).

Typical of many hypersaline environments, the halotolerant unicellular green alga Dunaliella (Chlorophyta) is implicated as the major primary producer (Wright and Burton, 1981). However, productivity measured in the lake is extremely low $\left(<10 \mathrm{~g} \mathrm{Cm}^{-2}\right.$ per year) (Campbell, 1978; Gibson, 1999). In addition to hypersalinity and seasonal extremes in light availability, low productivity has been attributed to a deficiency in available nutrients and the depth of the lake $(\sim 36 \mathrm{~m})$ preserving very low annual temperatures (for example, compared with shallower hypersaline Antarctic lakes) (Campbell, 1978; Wright and Burton, 1981; Ferris and Burton, 1988).

Based on the analysis of Deep Lake metagenome data, it was recently shown that while the lake community is dominated by heterotrophic halophilic archaea (haloarchaea) of the family Halobacteriaceae, the community composition differs 
greatly to temperate and tropical hypersaline environments (DeMaere et al., 2013). The community was found to have low complexity and be hierarchically structured. Genome sequences were obtained for four isolates: Halorubrum lacusprofundi strain ACAM34 (Franzmann et al., 1988), strain tADL (Candidatus Halohasta litchfieldiae; Mou et al., 2012), strain DL31 and strain DL1 (Halobacterium sp.), which represent phylogenetically distinct ( $\sim 85 \%$ 16S rRNA gene similarity) lineages. Based on small subunit rRNA pyrotag sequence data, the distribution of these four haloarchaea was found to be reasonably uniform throughout the water column, and to account for a total $\sim 72 \%$ of all pyrotags. However, the relative proportion of the four haloarchaea varied considerably, with tADL being the most abundant (comprising $\sim 44 \%$ of pyrotags), DL31 $\sim 18 \%$, H. lacusprofundi $\sim 10 \%$ and DL1 only $\sim 0.3 \%$.

A remarkable feature of the Deep Lake system was an observed high level of inter-genera gene exchange between tADL, DL31, H. lacusprofundi and DL1, including the sharing of long regions (up to $35 \mathrm{~kb}$ ) of virtually identical $(\sim 100 \%)$ DNA (DeMaere et al., 2013). Given the nature and extent of gene exchange, and a very low predicted rate of cell division of $\leqslant 6$ rounds per year (and hence vertical inheritance), a notable feature of the lake system was the maintenance of sympatric speciation (that is, distinct Halobacteriaceae lineages). Genetic differences that describe ecotypes with a capacity to perform niche adaptation would promote sympatric speciation, and consistent with this, preliminary genomic traits suggestive of physiological distinction were noted between the four Deep Lake haloarchaea (DeMaere et al., 2013).

In order to advance our understanding of the potential for niche differentiation, in the present study, we performed an in-depth comparative genomic analysis of the four Deep Lake haloarchaea and assessed functional properties through substrate growth studies. A focus was placed on elucidating characteristics of tADL that promote its dominance. By inferring apparent physiological differences, and linking this to physicochemical characteristics of the lake, we were able to better define ecotype distinctions and overall lake ecology.

\section{Materials and methods}

Strains and genomic analyses

Deep Lake water samples from 5, 13, 24 and $36 \mathrm{~m}$ depths were sequentially size fractionated through a $20-\mu \mathrm{m}$ prefilter onto $3.0,0.8$ and $0.1 \mu \mathrm{m}$ pore size, $293 \mathrm{~mm}$ polyethersulfone membrane filters (DeMaere et al., 2013). Isolation of strains and DNA sequencing of genomes and metagenomes have been described (DeMaere et al., 2013). Pyrosequencing of PCR-amplified V6-V8 region of small subunit rRNA genes was used to generate microbial community profiles, with operational taxonomic units (OTUs) defined based on 97\% sequence identity, as described previously (DeMaere et al., 2013). COG (Clusters of Orthologous Groups) scrambler and ALL scrambler were used to assess the statistical representation of COGs categories and individual COGs between the four Deep Lake haloarchaeal genomes, as described previously (Allen et al., 2009). For each comparison, the test genome was compared against the other three Deep Lake genomes, with subsample size of 10000 , confidence level 0.99 and 10000 bootstrap replicates. Genomes were interrogated for the presence or absence of genes that encode proteins and metabolic pathways that may provide ecophysiological distinctions between the four Deep Lake haloarchaea. To assess the veracity of gene functional assignments, all annotations of genes involved in pathways described here were manually examined as described previously (Allen et al., 2009).

\section{Growth studies}

The four Deep Lake haloarchaea, tADL, DL31, $H$. lacusprofundi and DL1 were tested for growth using potential carbon and nitrogen sources in batch cultures based on DBCM2 media (Burns and DyallSmith, 2006). DBCM2 contains low concentrations of peptone $(0.025 \% \mathrm{w} / \mathrm{v})$ and yeast extract $(0.005 \% \mathrm{w} / \mathrm{v})$, and growth of cultures did not occur unless these components were present in the media. Because essentially no change in turbidity occurred when strains were grown in unsupplemented DBCM2 media (data not shown), it is likely that the peptone and/or yeast extract provided essential nutrients, such as cofactors. Growth was assessed as maximum turbidity (optical density at $600 \mathrm{~nm}$ ) achieved for cultures grown aerobically in liquid broth $(20 \mathrm{ml}$ volume, 150 r.p.m., $30^{\circ} \mathrm{C}$ ), as described previously (Franzmann et al., 1988). Growth was evaluated against a blank where each strain was grown in DBCM2 without the addition of the defined substrates. The following defined carbon and/or nitrogen sources were tested (all at $10 \mathrm{~mm}$ ): glycerol, urea, sucrose, glucose, fructose, mannose, acetate, citrate, succinate or putrescine. These were tested alone or in combination with pyruvate $(10 \mathrm{~mm})$, except for succinate and citrate where pyruvate was not added. For growth assessments of carbon sources, ammonia was used as the defined nitrogen source. For growth with urea, urea was tested as a carbon source with ammonia, and as a nitrogen source with pyruvate. In addition, peptone was tested at $0.1 \%$, either alone or with pyruvate. Cultures grown on urea were tested for urease activity using a rapid urease test (Marshall et al., 1987).

\section{Analyses of lake ecology}

Ammonia, nitrate, nitrite, total nitrogen, dissolved reactive phosphorus, total phosphorus, total organic 
carbon, total dissolved carbon, total sulfur and total dissolved sulfur for Deep Lake were determined by American Public Health Associations Standard Methods at Analytical Services, Tasmania, as described previously (Yau et al., 2013). Statistical analyses (Pearson Product Moment Correlation, ANOVA (analysis of variance) regression analysis for statistical significance) were performed to identify correlations between microbial community composition (OTUs by depth and filter size; Supplementary Table S1) and lake chemistry (Supplementary Table S2). The relative abundances of individual OTUs were compared with one another and also with physicochemical parameters measured at the same depths where the biomass was sampled.

\section{Results}

Genome analysis overview

A summary of basic genome characteristics including rRNA operons, origins of replication, prediction of proteins with transmembrane domains and secretion signals, is provided in Supplementary Information. Statistical evaluation of COGs between the four genomes was performed (Table 1; Supplementary Table S3), and the COG categories (Figure 1) and individual COGs (Table 1) with statistically significant over-representation or under-representation provided clues about the functional traits characteristic of each of the haloarchaea. This information guided an in-depth appraisal of gene composition, including the compilation of genes associated with ATP-binding cassette (ABC) transport systems (Supplementary Table S4) and other functions (Supplementary Table S5). Based on these analyses, the important functional characteristics of the Deep Lake haloarchaea were inferred (described below). A graphical representation of functional traits that are inferred to be most important to the four Deep Lake haloarchaea are portrayed in Figure 2.

\section{Primary transport}

ABC transporters drive the translocation of substrates across membranes by the hydrolysis of ATP. A complete ABC transporter for the uptake of substrates comprises ATP-binding and permease and substrate-binding lipoprotein components. Because haloarchaea typically do not use organic solutes to maintain osmotic balance (Oren, 1999), imported organic solutes such as simple sugars, glycerol and amino acids are presumably consumed as substrates. All genomes have genes for $A B C$ transporters that target carbohydrates, including those with the highest sequence identities to $A B C$ transporters for glycerol-3-phosphate, oligosaccharides or nucleosides. ABC transporters for glycerol-3phosphate (Ugp) or oligosaccharides such as maltose share a close relationship with each other, and also with multiple sugar ABC transporters (Msm), and therefore cannot be distinguished based on sequence alone. Nevertheless, broad specificity (including functional exchangeability) has been documented for Ugp and maltose ABC transporters, at least in bacteria (Hekstra and Tomassen, 1993), from which haloarchaeal ABC transporters are thought to have been derived (Nelson-Sathi et al., 2012). Each genome has a single set of genes for a nucleoside ABC transporter for importing exogenous nucleotide sources, as an alternative to nucleotide biosynthesis.

tADL has the highest number of putative carbohydrate ABC transporters, but has no identifiable ABC transporters for oligopeptides/dipeptides. In contrast, DL31 has only one identifiable carbohydrate ABC transporter, but at least 15 oligopeptides/dipeptide ABC transporters, which is more than the other Deep Lake haloarchaea. Consistent with the abundance of oligopeptides/dipeptide ABC transporters, the genome of DL31 encodes the highest number of aminopeptidases that target di- and oligopeptides, including members of peptidase families S9 and M28 (13 and 5, respectively), and the highest number of secreted proteases of the peptidase family S8/S35 (5), which includes halolysin. DL31 and DL1 each have a prolyl iminopeptidase gene (family S33) that is not found in the other two Deep Lake haloarchaea. Many of these peptidase genes in DL31 are located close to the genes for oligopeptides/dipeptide ABC transporters. ABC transporters for amino acids are present in the genomes, particularly those with the highest identities for branched-chain amino acid (BCAA) ABC transporters. For DL1, BCAA ABC transporter genes were the largest class of $\mathrm{ABC}$ transporters represented in the genome.

Phosphate ABC transporters are present across all four genomes, often occurring in multiple copies. In addition, tADL contains genes for a phosphonate ABC transporter. In bacteria these high-affinity phosphate or phosphonate uptake systems are induced under conditions of phosphate limitation (Gebhard et al., 2006). The same is likely to be true for Deep Lake haloarchaea, given the presence of a regulatory protein gene adjacent to the $\mathrm{ABC}$ transporter genes. All four genomes have homologs of a corrinoid ABC transporter gene identified in Halobacterium salinarum NRC-1 (Woodson et al., 2005), although it is not clear whether these transporters in Deep Lake haloarchaea are specific for corrinoids or iron (III), as these types share a high level of sequence identity.

\section{Secondary transport}

Haloarchaea use a bioenergetically efficient 'salt-in' strategy of importing $\mathrm{K}^{+}$and $\mathrm{Cl}^{-}$ions (see Rhodopsins below), and expelling $\mathrm{Na}^{+}$ions. The elevated intracellular $\mathrm{K}^{+}$concentration is 
Table 1 Selected cellular functions and individual COGs over- or under-represented in Deep Lake haloarchaeal genomes

\begin{tabular}{|c|c|c|c|c|}
\hline \multirow[t]{2}{*}{ Cellular function } & \multicolumn{4}{|c|}{ Strain } \\
\hline & $t A D L$ & DL31 & H. lac & DL1 \\
\hline \multicolumn{5}{|l|}{ Carbohydrate metabolism, including uptake and catabolism } \\
\hline COG0395 ABC-type sugar transport system, permease component & 0.16 & - & - & - \\
\hline COG3387 glucoamylase and related glycosyl hydrolases & 0.16 & - & - & -0.12 \\
\hline COG1080 phosphoenolpyruvate-protein kinase (PTS system EI component) & 0.14 & - & - & - \\
\hline COG1082 sugar phosphate isomerases/epimerases & 0.14 & -0.14 & - & - \\
\hline COG1175 ABC-type sugar transport systems, permease components & 0.14 & - & - & - \\
\hline COG1925 phosphotransferase system, HPr-related proteins & 0.14 & - & - & - \\
\hline COG0554 glycerol kinase & 0.12 & - & - & - \\
\hline COG0158 fructose-1,6-bisphosphatase & 0.11 & - & - & - \\
\hline COG0366 glycosidases & 0.11 & -0.10 & 0.10 & -0.10 \\
\hline COG0578 glycerol-3-phosphate dehydrogenase & 0.11 & - & - & - \\
\hline COG0647 predicted sugar phosphatases of the HAD superfamily & 0.09 & - & - & - \\
\hline COG3075 anaerobic glycerol-3-phosphate dehydrogenase & 0.09 & - & - & - \\
\hline COG4813 trehalose utilization protein & 0.09 & - & - & - \\
\hline \multicolumn{5}{|l|}{ Oligopeptide uptake and degradation } \\
\hline COG1404 subtilisin-like serine proteases & -0.12 & 0.21 & - & -0.12 \\
\hline COG2234 predicted aminopeptidases & -0.20 & 0.26 & - & - \\
\hline COG4608 ABC-type oligopeptide transport system, ATPase component & -0.22 & 0.38 & - & - \\
\hline COG1506 dipeptidyl aminopeptidases/acylaminoacyl-peptidases & -0.25 & 0.48 & -0.19 & - \\
\hline COG0747 ABC-type dipeptide transport system, periplasmic component & -0.37 & 0.56 & - & - \\
\hline COG1173 ABC-type dipeptide/oligopeptide/nickel transport systems, permease components & -0.46 & 0.61 & - & - \\
\hline COG0444 ABC-type dipeptide/oligopeptide/nickel transport system, ATPase component & -0.49 & 0.64 & - & - \\
\hline COG0601 ABC-type dipeptide/oligopeptide/nickel transport systems, permease components & -0.49 & 0.58 & - & - \\
\hline COG0006 Xaa-Pro aminopeptidase & - & 0.29 & - & - \\
\hline \multicolumn{5}{|l|}{ BCAA uptake and degradation } \\
\hline COG1071 OADHC, dehydrogenase (E1) component, eukaryotic type, alpha subunit & - & - & - & 0.31 \\
\hline COG0022 OADHC, dehydrogenase (E1) component, eukaryotic type, beta subunit & - & - & - & 0.20 \\
\hline COG0410 ABC-type BCAA transport systems, ATPase component & - & - & - & 0.19 \\
\hline COG0508 OADHC, dihydrolipoamide acyltransferase (E2) component, and related enzymes & - & - & - & 0.16 \\
\hline COG1296 predicted BCAA permease & - & - & - & 0.11 \\
\hline \multicolumn{5}{|l|}{ Carboxylate transport } \\
\hline COG2358 TRAP-type uncharacterized transport system, periplasmic component & - & - & 0.11 & - \\
\hline COG4666 TRAP-type uncharacterized transport system, fused permease components & - & - & 0.11 & - \\
\hline COG1301 $\mathrm{Na}^{+} / \mathrm{H}^{+}$-dicarboxylate symporters & - & - & 0.10 & - \\
\hline COG1620 L-lactate permease & - & - & 0.08 & - \\
\hline \multicolumn{5}{|l|}{ Motility } \\
\hline COG1681 archaeal flagellins & 0.30 & -0.19 & - & - \\
\hline
\end{tabular}

Abbreviations: ABC, ATP-binding cassette; BCAA, branched-chain amino acid; COG, Clusters of Orthologous Groups; HAD, haloacid dehalogenase-like; H. lac, H. lacusprofundi; OADHC, 2-oxoacid dehydrogenase complex; PTS, phosphotransferase system; TRAP, tripartite ATP-independent periplasmic. Strains are tADL, DL31, H. lac and DL1.

maintained by active import of $\mathrm{K}^{+}$(Oren, 2002). All four genomes have genes that encode low-affinity $\mathrm{K}^{+}$transporters (TrkAH) driven by membrane potential, but no genes are present for ATP-driven $\mathrm{K}^{+}$import systems (KdpABC). For $\mathrm{Na}^{+}$ efflux, there are genes for multiple orthologs in DL1, $H$. lacusprofundi and DL31 for the $\mathrm{NhaC} \mathrm{Na} / \mathrm{H}^{+}$ antiporter. All four have genes for the multicomponent Mnh $(=\mathrm{Mrp}) \mathrm{Na}^{+} / \mathrm{H}^{+}$antiporter (Swartz et al., 2005).

Other transporters use electrochemical gradients to import organic solutes. Genes for amino acid transporters of the amino acid-polyamine-organocation (APC) superfamily are present in all four genomes, and most encode UspA domains and/or are adjacent to a UspA domain protein, as has been observed in other haloarchaeal genomes (Anderson et al., 2011).
The UspA domains are inferred to be involved in transporter regulation, although the precise mechanism is unclear (Schweikhard et al., 2010). All four genomes possess genes that encode phosphate permeases (sodium/phosphate symporters). These belong to the same family (PiT) as a sulfate permease from Gram-positive bacteria, and it is therefore possible that some of these PiT transporter genes are involved in sulfate rather than phosphate uptake in haloarchaea. Sulfate uptake implies a role in assimilatory sulfate reduction, a pathway that is currently not elucidated in haloarchaea (see Sulfur and phosphorus metabolism, below).

Unlike ABC transporters, tripartite ATP-independent periplasmic (TRAP) transporters utilize a proton motive force rather than ATP hydrolysis to drive the translocation of substrates (usually 


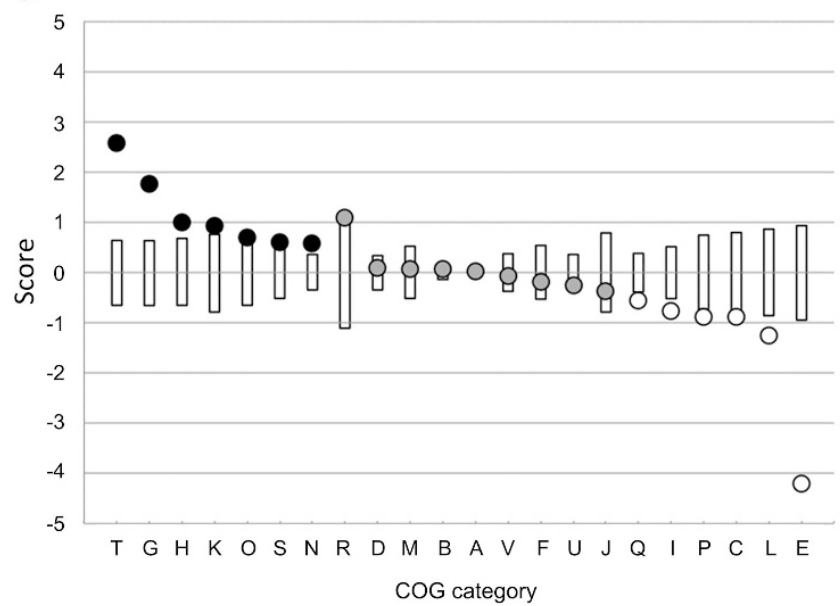

C

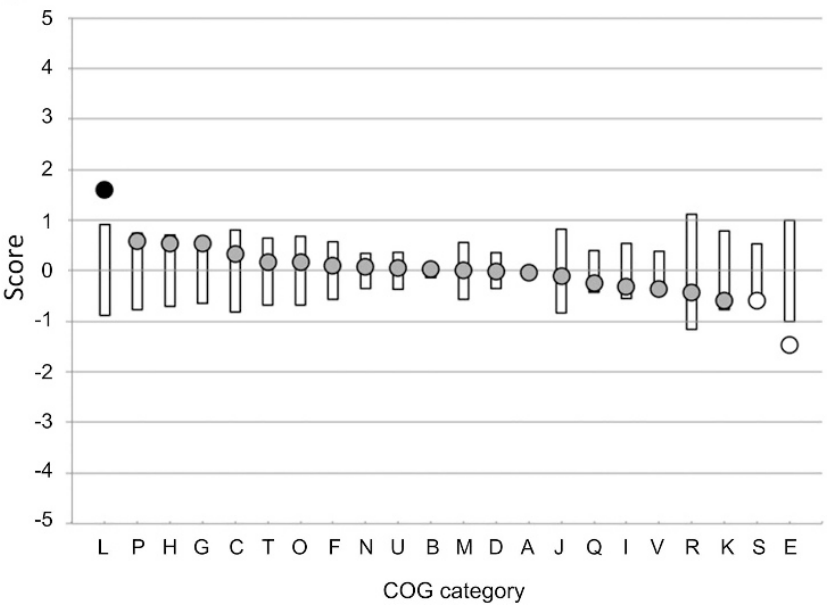

b

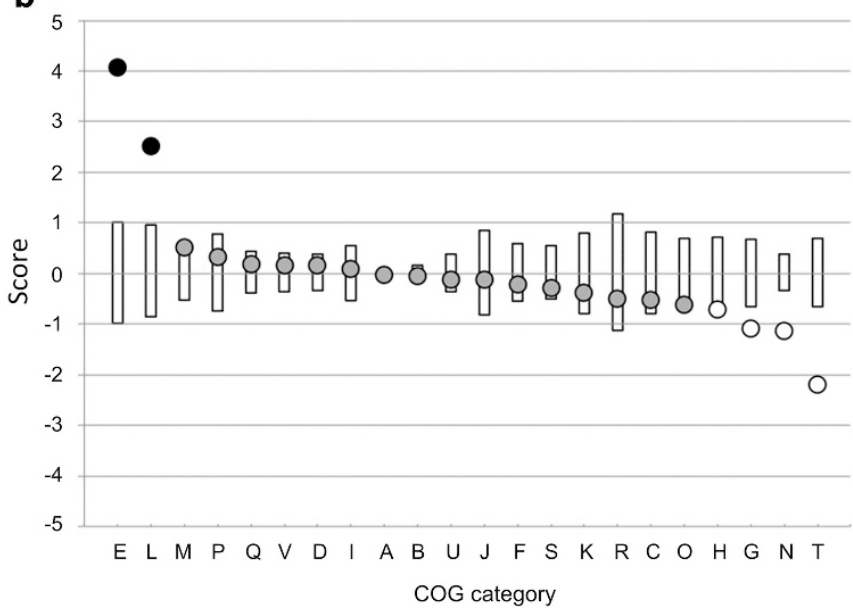

d

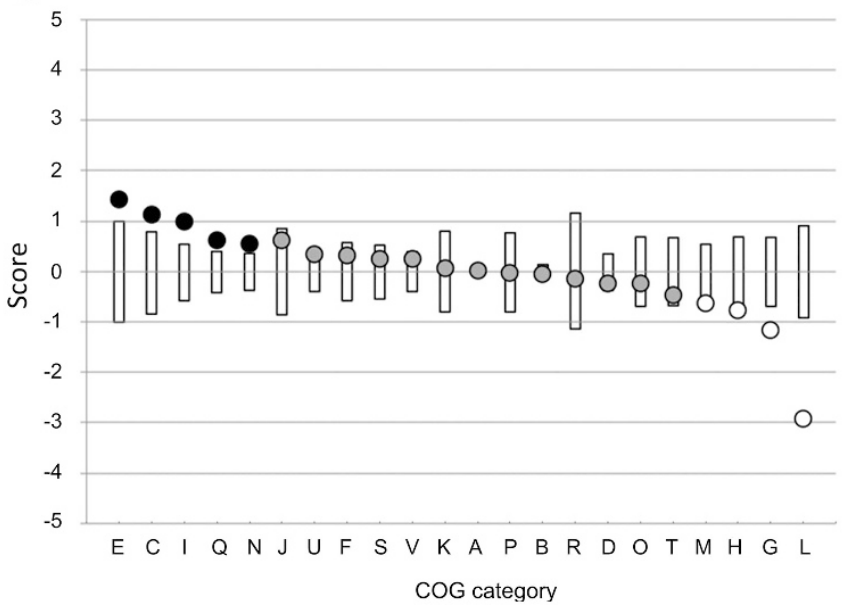

Figure 1 COGs category abundance in the genomes of Antarctic haloarchaea. (a) tADL, (b) DL31, (c) H. lacusprofundi and (d) DL1. Categories were determined to be significantly over-represented (black circle), significantly under-represented (white circle) or not statistically significant (gray circle) when compared with the other three Deep Lake genomes. White bars indicate 0.99 confidence interval. COGs category: [A] RNA processing and modification; [B] chromatin structure and dynamics; [C] energy production and conversion; [D] cell cycle control and mitosis; [E] amino acid transport and metabolism; [F] nucleotide metabolism and transport; [G] carbohydrate transport and metabolism; [H] coenzyme transport and metabolism; [I] lipid transport and metabolism; [J] translation; [K] transcription; [L] replication; recombination and repair; [M] cell wall/membrane/envelope biogenesis; [N] cell motility; [O] posttranslational modification, protein turnover and chaperones; [P] inorganic ion transport and metabolism; [Q] secondary metabolites biosynthesis, transport and catabolism; [R] general function prediction only; [S] function unknown; [T] signal transduction mechanisms; [U] intracellular trafficking, secretion and vesicular transport; [V] defense mechanisms.

carboxylic acids) into the cytoplasm (Forward et al., 1997). H. lacusprofundi has the highest number of TRAP transporter genes, and DL31 has none. $H$. lacusprofundi appears to lack symporters for the coupled uptake of sodium ions and di/tricarboxylic acids, although these are encoded in the genomes of the other three Deep Lake haloarchaea.

\section{Carbohydrate metabolism}

Glycerol may enter haloarchaeal cells by passive diffusion (Bolhuis et al., 2006), although a new family of glycerol transporters has been proposed to be present in haloarchaea (Anderson et al., 2011). Genes for the putative glycerol transporters are present in tADL and $H$. lacusprofundi, adjacent to genes for glycerol catabolism (Supplementary Table S5). Once in the cell, glycerol can be phosphorylated to glycerol-3-phosphate and then directed to glycolysis. A gene for glycerol kinase is present in H. lacusprofundi and DL31, and tADL has three orthologous glycerol kinase genes. All four genomes have genes for glycerol-3-phosphate dehydrogenase, which allows glycerol-3-phosphate to be oxidized to dihydroxyacetone phosphate (DHAP), and thereby enter glycolysis or gluconeogenesis. Genes for glycerol dehydrogenase and dihydroxyacetone kinase are present in tADL and $H$. lacusprofundi along with genes for a phosphoenolpyruvate (PEP)-dependent phosphotransferase system (PEP-PTS) that involves the cytosolic phosphorylation of dihydroxyacetone to DHAP by 


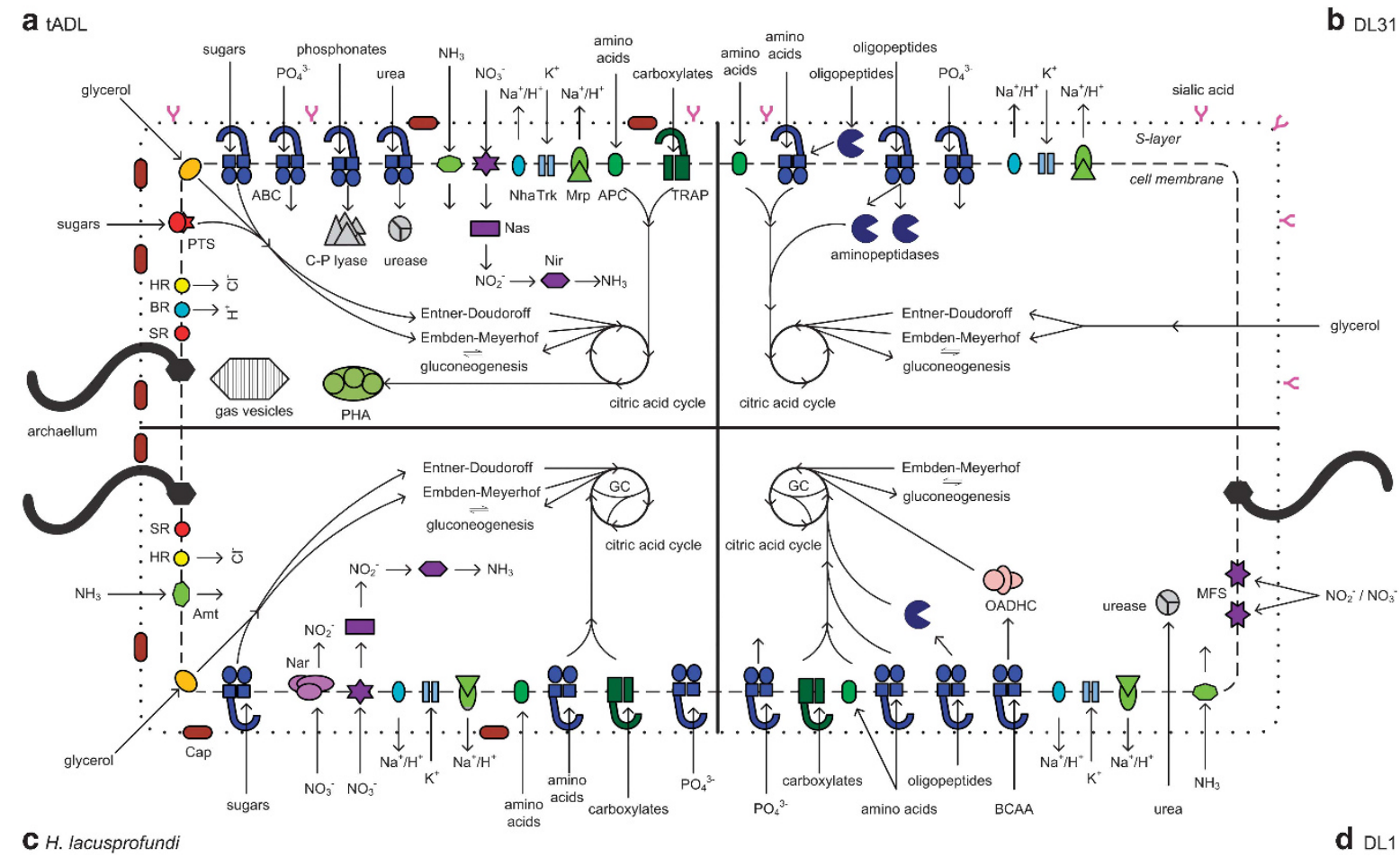

Figure 2 An integrated view of the ecophysiologies of the Antarctic haloarchaea. (a) DL31, (b) tADL, (c) H. lacusprofundi and (d) DL1. Cellular traits inferred to be important for survival in Deep Lake are shown, with depictions highlighting energy production, nutrient uptake, motility and osmotic balance. ABC, ATP-binding cassette transporter; Amt, ammonia transporter; APC, acid-polyamineorganocation transporter; BR, bacteriorhodopsin; C-P lyase, phosphonate degradation complex; Cap, poly-gamma-glutamate capsule; GC, glyoxylate cycle; HR, halorhodopsin; MFS, Major Facilitator Superfamily transporter; Mrp, multi-component $\mathrm{Na}^{+} / \mathrm{H}^{+}$antiporter; Nar, respiratory nitrate reductase; Nas, assimilatory nitrate reductase; Nha, $\mathrm{Na}^{+} / \mathrm{H}^{+}$antiporter; Nir, assimilatory nitrite reductase; OADHC, 2-oxoacid dehydrogenase complex; PHA, polyhydroxyalkanoate; PTS, phosphotransferase system; SR, sensory rhodopsin; TRAP, tripartite ATP-independent periplasmic transporter; Trk, low-affinity $\mathrm{K}^{+}$transporter.

dihydroxyacetone kinase (Bolhuis et al., 2006). DHAP is also a precursor to glycerol-1-phosphate, for construction of the glycerophosphate backbone of membrane phospholipids, and a gene for glycerol1-phosphate dehydrogenase was identified in all four genomes. There is no evidence in any of the four genomes of a dephosphorylating glycerol-3phosphate phosphatase for the direct liberation of phosphate.

The sugar PEP-PTS catalyzes the concomitant uptake and phosphorylation of sugars, in the first step in the breakdown of the imported sugar to pyruvate. tADL has two bacterial-type PEP-PTS gene clusters for sugar uptake, each of which encodes separate PEP-protein kinase (E1), phosphocarrier Hpr and enzyme II components (EIIA, EIIB and EIIC). In Haloferax volcanii, this bacterial-type PTS is used for the uptake and phosphorylation of fructose (Pickl et al., 2012). In tADL, each PTS gene cluster is adjacent to genes encoding enzymes involved in the modified Embden-Meyerhof pathway of glycolysis.

All four genomes have genes for the EmdenMeyerhof pathway for the breakdown of glucose to pyruvate, which is initiated by a broad-specificity hexokinase. tADL, DL31 and H. lacusprofundi also have genes for a modified (semiphosphorylated) Entner-Doudoroff pathway by which glucose is first oxidized to gluconate, and the gluconate is further converted to glyceraldehyde-3-phosphate and pyruvate via the intermediates 2-dehydro-3-deoxygluconate and 2-dehydro-3-deoxygluconate 6-phosphate (Danson et al., 2007; Falb et al., 2008). Genes responsible for the remaining steps in the glycolytic pathway (catabolism of glyceraldehyde-3-phosphate to pyruvate) are present in the four genomes. Under the growth conditions tested, only tADL and $H$. lacusprofundi showed abilities to grow on sugars as the sole defined carbon source (Supplementary Table S6), although both DL31 and DL1 have genes that encode putative carbohydrate uptake (ABC transporters) and metabolism genes (Tables S4 and S5). The presence of carbohydrate uptake and metabolism genes in haloarchaea that are reported not to grow on sugars has been observed previously (Ng et al., 2000), and may reflect potential saccharolytic abilities. Both tADL and $H$. lacusprofundi also have $\alpha$-amylase genes, although all four Deep Lake genomes lack genes for enzymes (for example, xylanase and chitinase) that are required for the degradation of plant or fungal biomass. All four genomes also have genes for a complete 
gluconeogenesis pathway, allowing amino acids to be used as sole carbon sources (Ng et al., 2000; Danson et al., 2007).

There are no genes associated with glycogen biosynthesis. Polyhydroxyalkanoate is accumulated by certain haloarchaea as a carbon and energy source, and can be sourced from unrelated precursors, including carbohydrates (Lu et al., 2008). tADL is the only one of the four Deep Lake haloarchaea to have genes for the polyhydroxyalkanoate biosynthetic pathway.

\section{Citric acid cycle (oxidative)}

Consistent with the heterotrophic metabolism of all four Deep Lake haloarchaea, all genes in the oxidative citric acid cycle were present in each genome. Although all four genomes have genes for at least two orthologs of acetyl-CoA synthetase, genes for enzymes of the glyoxylate cycle, which allows acetate to be used as the sole carbon source, were identified only in DL1 (isocitrate lyase and archaealtype malate synthase). $H$. lacusprofundi has a gene for isocitrate lyase, but not for malate synthase, as previously observed (Khomyakova et al., 2011). None of the four genomes has genes associated with the methylaspartate cycle, which is used for acetate assimilation in certain haloarchaea (Khomyakova et al., 2011).

\section{Amino acids and 2-oxoacids}

All four Deep Lake haloarchaea have the genomic capacity to biosynthesize the 20 amino acids necessary for protein biosynthesis. However, the presence of alternative pathways for amino acid biosynthesis varied between organisms. For example, whereas all four genomes have the potential to generate proline directly from ornithine, typical of haloarchaea, only tADL has a pathway predicted to derive proline from glutamate. The likely biosynthetic pathway for cysteine is clear in the DL1 and tADL genomes, deriving cysteine via activation of serine with acetyl-CoA, with acetylserine subsequently sulfhydrylated with sulfide. But in DL31 and $H$. lacusprofundi, the only available pathway is via a phosphoserine intermediate (generated in an earlier step of serine biosynthesis) that is sulfhydrylated using a cysteine synthase homolog, of which there are multiple copies in each genome. In all cases, the source of sulfide is unclear (see Sulfur and phosphorus metabolism below).

The capacity for degrading amino acids is encoded by all four genomes, but the ability to catabolize individual amino acids varies among the four Deep Lake haloarchaea. For example, DL1 is the only one of the four that has genes for the degradation of histidine (via urocanate) and tryptophan (to indole, or anthranilate via kynurenine). All Deep Lake genomes possess 2-oxoacid dehydrogenase complex (OADHC) gene clusters; DL1 has four, compared with one each in the other three genomes. Deamination of BCAAs generates branched-chain 2-oxoacids that can be further catabolized to citric acid cycle intermediates. However, none of the Deep Lake haloarchaea possess an identifiable arginine deiminase gene, indicating that none of them are capable of anaerobic growth by arginine fermentation (Dundas and Halvorson, 1966).

\section{Nitrogen metabolism}

Ammonia transporter genes are present in tADL, DL1 and $H$. lacusprofundi, but not in DL31. For ammonia assimilation, all genomes possess genes for glutamine synthetase, glutamate synthase and glutamate dehydrogenase. tADL also has two genes for the PII protein GlnK that are adjacent to each other and the ammonia transporter gene. In Haloferax mediterranei, both PII proteins have been shown to increase glutamine synthetase activity in the presence of 2-oxoglutarate (Pedro-Roig et al., 2013).

There was no genomic evidence that any of the four Deep Lake haloarchaea are capable of fixing dinitrogen. tADL and $H$. lacusprofundi each have a single gene for a nitrate/nitrite transporter of the Major Facilitator Superfamily family, whereas DL1 has four. Genes that encode proteins involved in assimilatory nitrate reduction were located only in $H$. lacusprofundi and tADL genomes, including ferredoxin-dependent assimilatory nitrate and nitrite reductases (Nas and Nir, respectively).

There is evidence for a denitrification capacity among the four Deep Lake haloarchaeal genomes. $H$. lacusprofundi has a gene cluster encoding a putative respiratory nitrate reductase (narGHI) and an enzyme chaperone (nar), suggesting that $H$. lacusprofundi might have the capacity to reduce nitrate to nitrite. Multicopper oxidase permease genes are present in the genomes of $H$. lacusprofundi, DL1 and tADL, and might represent nitrite reductase (nirK). Putative nitric oxide reductase (norB) genes are present in $H$. lacusprofundi and tADL, and putative nitrous oxide reductase (nosZ) genes are present in H. lacusprofundi, DL31 and DL1.

The tADL genome is the only one possessing a gene cluster for a urea $A B C$ transporter. Near the urea $\mathrm{ABC}$ transporter gene cluster is a urease gene cluster that encodes a cytoplasmic urease complex that hydrolyzes urea to ammonia and carbon dioxide. The DL1 genome also has a urease gene cluster, but no identifiable urea transporter genes. Genes for urea amidolyase were absent from all four Deep Lake genomes.

\section{Sulfur and phosphorus metabolism}

The biosynthesis of reduced sulfur (hydrogen sulfide) has not been experimentally characterized in haloarchaea. A proposed pathway involves the direct reduction of sulfate to sulfite by sulfite 
oxidoreductase, followed by reduction of sulfite to sulfide by sulfite reductase (Feng et al., 2012). Genes encoding both of these enzymes were identified in the genomes of tADL, $H$. lacusprofundi and DL1, and a gene for sulfite oxidoreductase, but not sulfite reductase, was identified in DL31. All four genomes have genes for rhodanese (thiosulfate sulfurtransferase) for the conversion of thiosulfate to sulfite. It is possible that one of the rhodanese proteins encoded in the DL31 genome actually functions as a sulfite reductase. Alternatively, DL31 may be dependent on exogenous reduced sulfur (for example, from oligopeptides) for biosynthesis. Dimethylsulfoniopropionate transport or catabolism genes were not detected in any of the genomes, and Dunaliella from nearby Organic Lake has been reported not to produce dimethylsulfoniopropionate when grown in culture (Franzmann et al., 1987). Therefore, dimethylsulfoniopropionate does not appear to be a potential source of reduced sulfur for the Deep Lake haloarchea.

Only tADL possesses the genomic capacity for phosphonate degradation. The essential genes for the ATP-dependent degradation of phosphonate to cyclic ribose-phosphate (Kamat et al., 2011) are located within a gene cluster that also includes phosphonate ABC transporter genes (Supplementary Table S4).

\section{Rhodopsins}

Archaeal rhodopsins are light-responsive transmembrane proteins that are used as light-driven ion pumps or as signal receptors for phototaxis. Bacteriorhodopsin (BR) is a proton pump that uses light energy to create an electrochemical gradient for ATP production; halorhodopsin uses light energy to transport $\mathrm{Cl}^{-}$ions into the cytoplasm, assisting the cell in maintaining osmotic balance; sensory rhodopsins (SRs) mediate attraction to orange light (SRI) or avoidance of UV/blue light (SRII) (Spudich, 2006; Sharma et al., 2007). Based on sequence identities (and conserved functional residues), only tADL possesses $\mathrm{BR}$, whereas only tADL and $H$. lacusprofundi possess halorhodopsin and SRII genes. For the latter SR, genes for the cognate transducers (HtrII) were also present.

\section{Motility: flagella and gas vesicles}

tADL, DL1 and $H$. lacusprofundi all contain gene clusters for flagellum biosynthesis (=archaellum; Jarrell and Albers, 2012). In these cases, the flagellar gene cluster is adjacent to one or more genes for chemotaxis proteins. However, only tADL possesses genes for gas vesicle biosynthesis. Gas vesicles are hollow, gas-filled structures made of protein that confer buoyancy to planktonic cells, allowing more efficient vertical migrations than swimming by flagella, particularly for slow-growing organisms (Walsby, 1994), and a function in combatting osmotic stress, or orientating cells positioned at the water surface, has also been proposed (reviewed in Pfeifer, 2012). Multiple GvpA orthologs, which function in the construction of the gas vesicle wall, are encoded, but GvpC, which strengthens the gas vesicle wall, or the regulatory proteins $\mathrm{GvpD}$ and GvpE (Walsby, 1994; Oren et al., 2005) were not identified in the tADL genome, as is the case for many other haloarchaea (Pfeifer, 2012). Having multiple copies of gvpA and lacking $g v p C$ and $g_{v p D E}$ is characteristic of certain methanogenic archaea that produce gas vesicles (Pfeifer, 2012). Halobacterium salinarum $\triangle g v p C$ deletion mutants are still capable of producing functional gas vesicles, albeit these are more fragile and irregularly shaped compared with wild-type cells (Offner et al., 1996), indicating that tADL may produce an unusual form of gas vesicles.

\section{Cell envelope}

Haloarchaeal surface layer glycoproteins can undergo both $\mathrm{N}$ - and O-glycosylation (reviewed in Eichler, 2003). The presence of a gene for the oligosaccharyltransferase AglB, plus multiple genes for glycosyltransferases, indicates that N-glycosylation pathways function in all four Deep Lake haloarchaea. The glycosyltransferase genes vary between the four genomes, indicating that the profiles of N-linked glycans would differ in these haloarchaea as has been observed between other species of haloarchaea (Kaminski et al., 2013). Genes for a bacterial-type CapBCA biosynthesis complex are encoded in tADL and $H$. lacusprofundi, indicating that they may be able to synthesize a poly-gamma-glutamate capsule (Ashiuchi and Misono, 2002). tADL and DL31 also have gene clusters associated with the construction of capsular polysaccharide using nucleotide sugars. In addition, the tADL genome encodes a halomucin homolog (Bolhuis et al., 2006). The presence of neuA and $n e u B$ genes in tADL and DL31 indicates that they can probably synthesize N-acetylneuraminic acid (sialic acid).

\section{Evaluation of growth properties}

To assess specific metabolic properties inferred from the genome sequences, growth studies were performed on the Deep Lake haloarchaea (Supplementary Table S6). All grew using pyruvate as the defined carbon and energy source, which is a common feature of haloarchaea. tADL and $H$. lacusprofundi were able to use simple sugars as defined carbon and energy sources, but DL31 and DL1 could not. Thus, only tADL and H. lacusprofundi can be described as being saccharolytic. The strongest growth of tADL was using a combination of pyruvate and sucrose or pyruvate and glycerol. However, tADL growth on sucrose as the defined carbon and energy source was weak. Initially, no growth was detectable on glycerol as the defined 
carbon and energy source, but when tADL grown on medium containing both pyruvate and glycerol was then transferred to medium containing just glycerol, growth was observed.

For DL31 and DL1, pyruvate was the only substrate that served as a defined carbon and energy source. Growth of both strains was enhanced in media supplemented with $0.1 \%$ peptone. Overall, $H$. lacusprofundi had the ability to grow on the widest range of substrates.

Urea was used as a nitrogen source by tADL and $H$. lacusprofundi. Urea-grown tADL cultures were strongly urease positive, whereas urea-grown $H$. lacusprofundi cultures were urease negative.

\section{Ecology inferred from physicochemical data}

Although Deep Lake is clearly dominated by haloarchaea, OTUs for bacteria were present, including those for Bacteroidetes, Gammaproteobacteria, Betaproteobacteria and Firmicutes (Supplementary Table S1). Within Gammaproteobacteria, a subset of OTUs matched Ectothiorhodospiraceae (purple sulfur bacteria), which suggests the presence of a potentially photoautotrophic bacterium in Deep Lake, although its low abundance $(0.6 \%)$ indicates that it would make a negligible contribution to primary productivity. The relative abundances of tADL and DL31 were negatively correlated (Pearson correlation coefficient $r=-0.82 ; P<0.01$ ), and DL31 was negatively correlated with Dunaliella $(r=-0.65 ; P<0.05)$. The relative abundance of DL1 was positively correlated with OTUs for the anaerobe Halanaerobium $(\sim 0.9 \%$ abundance; $r=0.65 ; P<0.01)$ and total Firmicutes $(\sim 1.2 \%$ abundance; $r=0.68 ; P<0.01)$. The relative abundance of DL31 also positively correlated with total organic carbon concentrations $(r=1.00$; $P<0.01)$ and with OTUs for the low abundance $(\sim 0.8 \%)$ gammaproteobacterium Halomonas subglaciescola $(r=0.95 ; P=0.05)$. The relative abundance for $\mathrm{Hm}$. subglaciescola positively correlated with nitrate concentration $(r=0.96 ; P<0.05)$. Dunaliella relative abundance positively correlated with the depth of the water column $(r=1.0 ; P<0.01$, for both chloroplast and 18S rRNA genes), and Dunaliella chloroplast and 18S rRNA gene relative abundances were also positively correlated $(r=1.0$; $P=0.01)$.

\section{Discussion}

Integration of genomic characteristics, growth properties, analysis of haloarchaeal distribution by filter size and depth, and assessment of lake chemistry enabled inferences to be drawn about the metabolic capacities and specific ecophysiological traits of the four Deep Lake haloarchaea. The distinctive features provided insights into substrate preferences occurring in the lake, how the four Deep Lake haloarchaea co-exist in the lake and why tADL is dominant. Below we discuss key aspects of their inferred ecophysiologies.

$t A D L$

We identified a number of reasons that may explain the numerical dominance of tADL in Deep Lake. Of the four Deep Lake haloarchaea, only tADL possesses genes for BR and gas vesicles. The use of the latter as flotation devices facilitates vertical migration and enables access to solar irradiation at the lake surface by membrane-bound BR. Although the distribution of tADL within Deep Lake is generally homogeneous, it is less abundant at lower depths (DeMare et al., 2013; Supplementary Table S1). Gas vesicle formation in tADL may therefore be linked to photoheterotrophic growth. This may particularly boost tADL numbers in summer when illumination and algal primary production are highest.

The halotolerant microalga Dunaliella accumulates molar concentrations of glycerol as a compatible solute for osmotic stabilization (Borowitzka, 1981). Although the cytoplasmic membrane of Dunaliella is far less permeable to glycerol than that of other algae, leakage of glycerol does occur (Bardavid et al., 2008), and glycerol will also be released because of cell death and lysis. Glycerol is therefore regarded as a major carbon and energy source for haloarchaea in hypersaline habitats (Bardavid et al., 2008; Sherwood et al., 2009), including Deep Lake (Franzmann et al., 1988). Of the four Deep Lake genomes, only DL1 lacks genes for enzymes required for glycerol catabolism. Overall, tADL and $H$. lacusprofundi appear to be best equipped to utilize glycerol as a carbon and energy source in Deep Lake: both have dual pathways for glycerol degradation to DHAP (initiated by glycerol kinase or glycerol dehydrogenase), and tADL has genes for multiple orthologs of glycerol kinase and a putative membrane protein implicated in glycerol uptake (Anderson et al., 2011). Growth studies confirmed the ability of both tADL and $H$. lacusprofundi to utilize glycerol, with especially strong growth observed for tADL when both glycerol and pyruvate were provided in the medium (Supplementary Table S6).

The genomic evidence indicates that tADL has a 'sweet tooth', with a highly saccharolytic metabolism served by abundant ABC transporters and regulation by PTS. Although both tADL and $H$. lacusprofundi are saccharolytic, only the tADL genome is statistically over-represented for the COGs category associated with carbohydrate transport and metabolism (Figure 1), and for individual COGs associated with carbohydrate uptake and metabolism (Table 1, Supplementary Table S3). PTS indicates that catabolite repression occurs, allowing a rapid response to preferred carbon and energy sources. Nevertheless, as with glycerol, our growth assays indicate that growth on sucrose 
and mannose is enhanced in the presence of pyruvate. Surplus carbon could be stored by tADL as polyhydroxyalkanoate and mobilized under carbon-depleted conditions. A preference for highenergy substrates could also permit the bioenergetic investment required to degrade phosphonates. Phosphonates are abundant in nature (for example, as components of lipids), but the ability to use phosphonates as a substrate requires cleavage of the strong $\mathrm{C}-\mathrm{P}$ bond.

In addition to the above, the tADL genome is the only one with genes for a nitrogen regulatory system (involving PII), thereby integrating responses to nitrogen and carbon availability (Pedro-Roig et al., 2013). tADL also appears to be able to source ammonia directly from the environment using specific transporters, as does $H$. lacusprofundi and DL1. Ammonia levels do not appear to be limiting in Deep Lake. Nevertheless, tADL and H. lacusprofundi have genomic evidence for nitrate uptake and assimilatory reduction. Nitrate was detected in Deep Lake, and possibly derives from bird guano (for example, penguins and skuas). tADL also potentially derives nitrogen from a diverse array of organic sources, including urea, free amino acids and polyamines. However, in contrast to DL31 (see DL31, below), tADL appears not to utilize oligopeptides (Table 1, Supplementary Table S3). Although urea can cross the cell membrane by passive diffusion, the genome of tADL encodes a urea ABC transporter system. The major source of urea in the Vestfold Hills is likely from pinniped excreta; by contrast, guano is dominated by uric acid and does not contain urea (Lindeboom, 1984). The ability of tADL to grow using urea as a nitrogen source is consistent with the presence of urea transport and urease genes and detectable urease activity.

\section{DL31}

All data point to DL31 having a heterotrophic strategy very different to tADL. DL31 is the only one that is lacking genes for swimming motility and/or gas vesicles. The genome (including COGs analyses) indicates a highly proteolytic metabolism, with abundant peptide transporters and proteolytic enzymes (Table 1, Supplementary Tables S3 and S5). The negative correlation between the distributions of tADL and DL31 across filter sizes at different depths (Supplementary Table S1) is likely to reflect divergent ecological strategies within the water column. One hypothesis is that DL31 adheres to particulate matter and targets proteinaceous substrates in detritus. This is consistent with the strong positive correlation between the relative abundance of DL31 and total organic carbon concentrations.

However, the data do not support an affinity for Dunaliella cells per se, as the relative abundance of DL31 was in fact inversely correlated with Dunaliella OTUs. Similarly, there was no correlation between OTUs for Halomonas and Dunaliella, even though a syntrophic relationship between the two taxa has been proposed, with Halomonas growing on dissolved organic carbon exuded by primary producing Dunaliella (Keshtacher-Liebson et al., 1995). The positive correlation observed between the abundances of DL31 and Halomonas might therefore suggest a similar substrate preference. Hm. subglaciescola is a non-saccharolytic halophile originally isolated from nearby Organic Lake, and this bacterium has been proposed to grow on proteinaceous compounds released from decaying penguin feathers molted during the summer (Franzmann et al., 1987). These data support a preference for detritus rather than living algal cells.

Unlike the other three Deep Lake haloarchaea, DL31 lacks genes for the transport of ammonia or nitrate/nitrite. The increased genomic capacity of DL31 for uptake of organic nitrogen compounds likely obviates the need for either ammonia uptake from the environment or assimilatory nitrate reduction. This physiological characteristic may also distinguish the ecological niches that DL31 is capable of colonizing.

DL31 has the genomic capacity to degrade glycerol (similar to tADL and H. lacusprofundi), although the initial step in DL31 can only be catalyzed by glycerol kinase. In the absence of any identifiable glycerol transporters in the genome, DL31 would be reliant upon passive diffusion for glycerol uptake. Generally, cell membranes are permeable to glycerol (Bardavid et al., 2008), so this could be sufficient for accessing this substrate, especially if glycerol is obtained by DL31 attachment to Dunaliella-derived cell debris.

\section{H. lacusprofundi}

Similar to tADL, $H$. lacusprofundi is capable of using a range of organic acids and simple sugars for growth (Franzmann et al., 1988). By inference of metabolic capacity and substrate preference, $H$. lacusprofundi is the least specialized of the four Deep Lake haloarchaea. This is consistent with it having the lowest number of over- or underrepresented metabolism-related COGs (Figure 1). The lack of specialization may explain why it is by far the most represented species arising from cultivation studies of Deep Lake water (Franzmann et al., 1988).

Beyond this general trait, a number of specific characteristics of $H$. lacusprofundi are worth noting. $H$. lacusprofundi has the highest number of TRAP transporters, indicating that it may prefer organic acids (Table 1; Supplementary Table S3). $H$. lacusprofundi is capable of growth on acetate (Franzmann et al., 1988), possibly by using a glyoxylate cycle that includes a novel malate synthase (Lynch et al., 2012). Similarly, H. lacusprofundi grew with urea as a sole nitrogen source, despite the genome lacking identifiable genes for 
urea transport or catabolism (urease and urea amidolyase) and cultures testing urease negative (see above; Franzmann et al., 1988).

A complete denitrification pathway was identified in the $H$. lacusprofundi genome, despite the original description of $H$. lacusprofundi describing its inability to perform nitrate respiration (Franzmann et al., 1988). Specific growth conditions may be required to promote nitrate respiration and other denitrifying activities. In this regard, it is noteworthy that despite overall low OTU abundance in the lake (Supplementary Table S1), the two most abundant bacteria, Halospina denitrificans and $\mathrm{Hm}$. subglaciescola, are both facultatively anaerobic Gammaproteobacteria that can reduce nitrate to nitrite and $\mathrm{N}_{2} \mathrm{O}$ (Franzmann et al., 1987; Sorokin et al., 2006). Nitrite and $\mathrm{N}_{2} \mathrm{O}$ intermediates of denitrification may serve as terminal electron acceptors for anaerobic growth by certain Deep Lake haloarchaea (possibly $H$. lacusprofundi), and dissimilatory nitrate reduction by halophilic bacteria and/or haloarchaea may contribute to the nitrite detected in Deep Lake (Supplementary Table S2).

\section{DL1}

Genomic evidence suggests that amino acids are preferred substrates for DL1, and that glycerol cannot be used. However, in common with the three other Deep Lake haloarchaea, DL1 has the genomic potential for the utilization of glycerol-3-phosphate (a degradation product of bacterial and eucaryal phospholipids). Amino acids are likely obtained by DL1 directly from the environment. However, although both DL1 and DL31 genomes were statistically over-represented for the COGs category related to amino acid metabolism (Figure 1), there was no genomic evidence that DL1 is specialized for the degradation of peptides. Unlike DL31, DL1 encodes a gene for ammonia permease. Degradation of amino acids by DL1 explains the presence of urease genes without genes for identifiable urea transporters, because urea is generated endogenously by degradation of arginine (Baliga et al., 2004). In addition, the DL1 genome was statistically overrepresented for COGs associated with BCAA uptake and catabolism, including OADHC components (Table 1, Supplementary Table S3). In archaea, certain OADHCs can catalyze the decarboxylation of branched-chain 2-oxoacids that result from BCAA catabolism, as well as pyruvate (Heath et al., 2004, 2007; Sisignano et al., 2010). Thus, these COGs associated with general amino acid metabolism, BCAA uptake and catabolism, and OADHC components, likely describe a common substrate preference by DL1.

DL1 is by far the least abundant of the four Deep Lake haloarchaea. This may relate to its metabolic preference for amino acids as carbon and energy sources combined with the inability to catabolize glycerol. Free amino acids may not be readily available in Deep Lake; the abilities of all four Deep Lake haloarchaea to biosynthesize the 20 proteinogenic amino acids are consistent with a nutrient-depleted environment and the extremely low productivity of Deep Lake (Campbell, 1978). The high number of nitrate/nitrite transporter and multicopper oxidase genes in DL1 might also indicate a capacity, and possibly a preference, for anaerobic growth via nitrite respiration; this property would be unlikely to promote competitiveness throughout the oxic water column, but may provide niche adaptation.

\section{Capsular polysaccharide biosynthesis}

tADL, DL31 and H. lacusprofundi have a genomic capacity for capsular polysaccharide biosynthesis. The production of extracellular polymeric substances (EPS) and biofilm formation has been observed for certain strains of tADL and $H$. lacusprofundi, with $\mathrm{N}$-acetylglucosamine and/or sialic acid identified as constituent glycoconjugates (Fröls et al., 2012). EPS promote cell aggregation and biofilm formation of archaea at low temperatures (Reid et al., 2006; Allen et al., 2009; Williams et al., 2011). As well as facilitating adhesion to solid surfaces, EPS is also inferred to serve in protection against desiccation, osmotic stress and phage attack (Sutherland, 2001). For the Deep Lake haloarchaea, the EPS may protect cells against dehydration, facilitate membrane function in the cold and also modulate viral infection; viruses have been detected and previously associated with gene transfer events between the Deep Lake haloarchaea (DeMaere et al., 2013). For non-motile DL31 cells, the EPS may have a particular role in particle attachment.

The presence of distinct glycosyltransferase genes and the specific complement of cell envelope genes in the four genomes indicate that the type of EPS produced is likely to vary between the Deep Lake haloarchaea. Futhermore, in tADL, the neuA and $n e u B$ genes involved in the synthesis of sialic acid reside in a gene cluster that has previously been suggested as a marker of genomic variation indicative of ecotypes; the region was found to have low fragment recruitment coverage of metagenome data, indicating that sub-populations with specific phenotypic differences occur in the lake (DeMaere et al., 2013).

\section{Conclusions}

Based on genomic comparisons, the four Antarctic haloarchaea examined showed distinct substrate preferences. tADL showed evidence of a 'highenergy' saccharolytic metabolism that complements the ability to utilize biogenergetically expensive substrates (for example, urea and phosphonate) and to construct gas vesicles to optimize light harvesting by BR at the lake surface. The extensive illumination 
of the lake surface during the Antarctic summer is likely to promote photoheterotrophic growth. $H$. lacusprofundi is also saccharolytic, but the genome suggests a more versatile heterotrophic metabolism than tADL and a higher reliance on organic acids as carbon sources. In contrast, DL31 and DL1 appear to be metabolic specialists that target peptides and free amino acids, respectively, with the former possibly associating with particulate organic matter. The data are also consistent with the hypothesis that a capacity to catabolize glycerol contributes toward the relative abundance of the four species, insofar as Deep Lake is a hypersaline environment where Dunaliella is the major primary producer. In general, the OTU and genomic data provide evidence for niche partitioning among Deep Lake haloarchaea. Coexistence of specialist and versatile haloarchaea has been proposed for other hypersaline environments (Javor, 1984). Further work on ecological interactions of the Deep Lake haloarchaeal community is currently being investigated using metaproteomics in order to determine whether the substrate preferences hypothesized for these four haloarchaea (tADL, DL31, H. lacusprofundi and DL1) are corroborated by protein abundance levels in Deep Lake.

\section{Conflict of Interest}

The authors declare no conflict of interest.

\section{Acknowledgements}

This work was supported by the Australian Research Council and the Australian Antarctic Science program and undertaken with the assistance of resources provided at the NCI National Facility systems at the Australian National University through the National Computational Merit Allocation Scheme supported by the Australian Government. The work conducted by the United States Department of Energy Joint Genome Institute is supported by the Office of Science of the United States Department of Energy under contract no. DE-AC02-05CH11231. We thank Bernhard Tschitschko for his assistance with growth studies. We warmly acknowledge the positive and constructive comments made by reviewers during the review process.

\section{References}

Allen MA, Lauro FM, Williams TJ, Burg D, Siddiqui KS, De Francisci D et al. (2009). The genome sequence of the psychrophilic archaeon, Methanococcoides burtonii: the role of genome evolution in cold adaptation. ISME J 3: 1012-1035.

Anderson I, Scheuner C, Göker M, Mavromatis K, Hooper SD, Porat I et al. (2011). Novel insights into the diversity of catabolic metabolism from ten haloarchaeal genomes. PLoS One 6: e20237.
Ashiuchi M, Misono H. (2002). Biochemistry and molecular genetics of poly-gamma-glutamate synthesis. Appl Microbiol Biotechnol 59: 9-14.

Baliga NS, Bonneau R, Facciotti MT, Pan M, Glusman G, Deutsch EW et al. (2004). Genome sequence of Haloarcula marismortui: a halophilic archaeon from the Dead Sea. Genome Res 14: 2221-2234.

Bardavid RE, Khristo P, Oren A. (2008). Interrelationships between Dunaliella and halophilic prokaryotes in saltern crystallizer ponds. Extremophiles 12: 5-14.

Bolhuis H, Palm P, Wende A, Falb M, Rampp M, Rodriguez-Valera $\mathrm{F}$ et al. (2006). The genome of the square archaeon Haloquadratum walsbyi: life at the limits of water activity. BMC Genomics 7: 169.

Borowitzka LJ. (1981). The microflora. Adaptations to life in extremely saline lakes. Hydrobiologia 81: 33-46.

Burns DG, Dyall Smith M. (2006). Cultivation of haloarchaea. Methods Microbiol 35: 535-552.

Campbell PJ. (1978). Primary productivity of a hypersaline Antarctic lake. Aust J Mar Freshw Res 29: 717-724.

Danson MJ, Lamble HJ, Hough DW. (2007). Central metabolism. In: Cavicchioli R (ed). Archaea: Molecular and Cellular Biology. American Society for Microbiology: Washington, DC, USA, pp 260-287.

DeMaere MZ, Williams TJ, Allen MA, Brown MV, Gibson JAE, Rich J et al. (2013). High level of intergenera gene exchange shapes the evolution of haloarchaea in an isolated Antarctic lake. Proc Natl Acad Sci 110: 16939-16944.

Dundas IE, Halvorson HO. (1966). Arginine metabolism in Halobacterium salinarium, an obligately halophilic bacterium. J Bacteriol 91: 113-119.

Eichler J. (2003). Facing extremes: archaeal surface-layer (glyco)proteins. Microbiology 149: 3347-3351.

Falb M, Müller K, Königsmaier L, Oberwinkler T, Horn P, von Gronau S et al. (2008). Metabolism of halophilic archaea. Extremophiles 12: 177-196.

Feng J, Liu B, Zhang Z, Ren Y, Li Y, Gan F et al. (2012). The complete genome sequence of Natrinema sp. J7-2, a haloarchaeon capable of growth on synthetic media without amino acid supplements. PLoS One 7: e41621.

Ferris JM, Burton HR. (1988). The annual cycle of heat content and mechanical stability of hypersaline Deep Lake, Vestfold Hills, Antarctica. Hydrobiologia 165: 115-128.

Forward JA, Behrendt MC, Wyborn NR, Cross R, Kelly DJ. (1997). TRAP transporters: a new family of periplasmic solute transport systems encoded by the $\operatorname{dct} P Q M$ genes of Rhodobacter capsulatus and by homologs in diverse gram-negative bacteria. J Bacteriol 179: 5482-5493.

Franzmann PD, Deprez PP, Burton HR, van den Hoff J. (1987). Limnology of Organic Lake, Antarctica, a meromictic lake that contains high concentrations of dimethyl sulfide. Aust J Mar Freshw Res 38: 409-417.

Franzmann PD, Stackebrandt E, Sanderson K, Volkman JK, Cameron DE, Stevenson PL et al. (1988). Halobacterium lacusprofundi, sp. nov., a halophilic bacterium isolated from Deep Lake, Antarctica. Syst Appl Microbiol 11: 20-27.

Fröls S, Dyall-Smith M, Pfeifer F. (2012). Biofilm formation by haloarchaea. Environ Microbiol 14: 3159-3174.

Gebhard S, Tran SL, Cook GM. (2006). The Phn system of Mycobacterium smegmatis: a second high-affinity ABC-transporter for phosphate. Microbiology 152: 3453-3465. 
Gibson JAE. (1999). The meromictic lakes and stratified marine basins of the Vestfold Hills, East Antarctica. Antarct Sci 11: 175-192.

Heath C, Jeffries AC, Hough DW, Danson MJ. (2004). Discovery of the catalytic function of a putative 2-oxoacid dehydrogenase multienzyme complex in the thermophilic archaeon Thermoplasma acidophilum. FEBS Lett 577: 523-527.

Heath C, Posner MG, Aass HC, Upadhyay A, Scott DJ, Hough DW et al. (2007). The 2-oxoacid dehydrogenase multi-enzyme complex of the archaeon Thermoplasma acidophilum - recombinant expression, assembly and characterization. FEBS J 274: 5406-5415.

Hekstra D, Tommassen J. (1993). Functional exchangeability of the ABC proteins of the periplasmic binding protein-dependent transport systems Ugp and Mal of Escherichia coli. J Bacteriol 175: 6546-6552.

Jarrell KF, Albers SV. (2012). The archaellum: an old motility structure with a new name. Trends Microbiol 20: $307-312$.

Javor BJ. (1984). Growth potential of halophilic bacteria isolated from solar salt environments: carbon sources and salt requirements. Appl Environ Microbiol 48: 352-360.

Kamat SS, Williams HJ, Raushel FM. (2011). Intermediates in the transformation of phosphonates to phosphate by bacteria. Nature 480: 570-573.

Kaminski L, Lurie-Weinberger MN, Allers T, Gophna U, Eichler J. (2013). Phylogenetic- and genome-derived insight into the evolution of N-glycosylation in Archaea. Mol Phylogenet Evol 68: 327-339.

Keshtacher-Liebson E, Hadar Y, Chen Y. (1995). Oligotrophic bacteria enhance algal growth under irondeficient conditions. Appl Environ Microbiol 61: 2439-2441.

Khomyakova M, Bükmez Ö, Thomas LK, Erb TJ, Berg IA. (2011). A methylaspartate cycle in haloarchaea. Science 331: 334-337.

Lindeboom HJ. (1984). The nitrogen pathway in a penguin rookery. Ecology 65: 269-277.

Lu Q, Han J, Zhou L, Zhou J, Xiang H. (2008). Genetic and biochemical characterization of the poly(3-hydroxybutyrate-co-3-hydroxyvalerate) synthase in Haloferax mediterranei. J Bacteriol 190: 4173-4180.

Lynch EA, Langille MG, Darling A, Wilbanks EG, Haltiner C, Shao KS et al. (2012). Sequencing of seven haloarchaeal genomes reveals patterns of genomic flux. PLoS One 7: e41389.

Marshall BJ, Warren JR, Francis GJ, Langton SR, Goodwin CS, Blincow ED. (1987). Rapid urease test in the management of Campylobacter pyloriassociated gastritis. Am J Gastroenterol 82: 200-210.

Mou YZ, Qiu XX, Zhao ML, Cui HL, Oh D, Dyall-Smith ML. (2012). Halohasta litorea gen. nov. sp. nov., and Halohasta litchfieldiae sp. nov., isolated from the Daliang aquaculture farm, China and from Deep Lake, Antarctica, respectively. Extremophiles 16: 895-901.

Nelson-Sathi S, Dagan T, Landan G, Janssen A, Steel M, McInerney JO et al. (2012). Acquisition of 1,000 eubacterial genes physiologically transformed a methanogen at the origin of Haloarchaea. Proc Natl Acad Sci USA 109: 20537-20542.

Ng WV, Kennedy SP, Mahairas GG, Berquist B, Pan M, Shukla HD et al. (2000). Genome sequence of Halobacterium species NRC-1. Proc Natl Acad Sci USA 97: 12176-12181.
Offner S, Wanner G, Pfeifer F. (1996). Functional studies of the gvpACNO operon of Halobacterium salinarium reveal that the GvpC protein shapes gas vesicles. J Bacteriol 178: 2071-2078.

Oren A. (1999). Bioenergetic aspects of halophilism. Microbiol Mol Biol Rev 63: 334-348.

Oren A. (2002). Diversity of halophilic microorganisms: environments, phylogeny, physiology, and applications. J Ind Microbiol Biotechnol 28: 56-63.

Oren A, Pri-El N, Shapiro O, Siboni N. (2005). Gas vesicles isolated from Halobacterium cells by lysis in hypotonic solution are structurally weakened. FEMS Microbiol Lett 252: 337-341.

Pedro-Roig L, Camacho M, Bonete MJ. (2013). Regulation of ammonium assimilation in Haloferax mediterranei: interaction between glutamine synthetase and two GlnK proteins. Biochim Biophys Acta 1834: 16-23.

Pfeifer F. (2012). Distribution, formation and regulation of gas vesicles. Nat Rev Microbiol 10: 705-715.

Pickl A, Johnsen U, Schönheit P. (2012). Fructose degradation in the haloarchaeon Haloferax volcanii involves a bacterial type phosphoenolpyruvate-dependent phosphotransferase system, fructose-1-phosphate kinase, and class II fructose-1,6-bisphosphate aldolase. J Bacteriol 194: 3088-3097.

Reid IN, Sparks WB, Lubow S, McGrath M, Livio M, Valenti J et al. (2006). Terrestrial models for extraterrestrial life: methanogens and halophiles at Martian temperatures. Intl J Astrobiol 5: 89-97.

Sharma AK, Walsh DA, Bapteste E, Rodriguez-Valera F, Ford Doolittle W, Papke RT. (2007). Evolution of rhodopsin ion pumps in haloarchaea. BMC Evol Biol 7: 79.

Schweikhard ES, Kuhlmann SI, Kunte HJ, Grammann K, Ziegler CM. (2010). Structure and function of the universal stress protein TeaD and its role in regulating the ectoine transporter TeaABC of Halomonas elongata DSM 2581 ${ }^{\mathrm{T}}$. Biochemistry 49: 2194-2204.

Sherwood KE, Cano DJ, Maupin-Furlow JA. (2009). Glycerol-mediated repression of glucose metabolism and glycerol kinase as the sole route of glycerol catabolism in the haloarchaeon Haloferax volcanii. J Bacteriol 191: 4307-4315.

Sisignano M, Morbitzer D, Gätgens J, Oldiges M, Soppa J. (2010). A 2-oxoacid dehydrogenase complex of Haloferax volcanii is essential for growth on isoleucine but not on other branched-chain amino acids. Microbiology 156: 521-529.

Sorokin DY, Tourova TP, Galinski EA, Belloch C, Tindall BJ. (2006). Extremely halophilic denitrifying bacteria from hypersaline inland lakes, Halovibrio denitrificans sp. nov. and Halospina denitrificans gen. nov., sp. nov., and evidence that the genus name Halovibrio Fendrich 1989 with the type species Halovibrio variabilis should be associated with DSM 3050. Int J Syst Evol Microbiol 56: 379-388.

Spudich JL. (2006). The multitalented microbial sensory rhodopsins. Trends Microbiol 14: 480-487.

Sutherland IW. (2001). Biofilm exopolysaccharides: a strong and sticky framework. Microbiology 147: 3-9.

Swartz TH, Ikewada S, Ishikawa O, Ito M, Krulwich TA. (2005). The Mrp system: a giant among monovalent cation/proton antiporters? Extremophiles 9: 345-354.

Walsby AE. (1994). Gas vesicles. Microbiol Rev 58: 94-144.

Wilkins D, Yau S, Williams TJ, Allen MA, Brown MV, DeMaere MZ et al. (2013). Key microbial drivers in Antarctic aquatic environments. FEMS Microbiol Rev 37: 303-335. 
Williams TJ, Lauro FM, Ertan H, Burg DW, Poljak A, Raftery MJ et al. (2011). Defining the response of a microorganism to temperatures that span its complete growth temperature range ( -2 to $\left.28^{\circ} \mathrm{C}\right)$ using multiplex quantitative proteomics. Environ Microbiol 13: 2186-2203.

Woodson JD, Reynolds AA, Escalante-Semerena JC. (2005). ABC transporter for corrinoids in Halobacterium sp. strain NRC-1. J Bacteriol 187: 5901-5909.
Wright SW, Burton HR. (1981). The biology of antarctic saline lakes. Hydrobiologia 82: 319-338.

Yau S, Lauro FM, Williams TJ, Demaere MZ, Brown MV, Rich J et al. (2013). Metagenomic insights into strategies of carbon conservation and unusual sulfur biogeochemistry in a hypersaline Antarctic lake. ISME J 7: 1944-1961.

Supplementary Information accompanies this paper on The ISME Journal website (http://www.nature.com/ismej) 